\title{
MOBILISING" WEB SITES AT AN OPEN UNIVERSITY: THE ATHABASCA UNIVERSITY EXPERIENCE
}

\author{
Rory McGreal \\ Athabasca University \\ rory@athabascau.ca \\ Regina Wasti \\ Independent \\ Regina.wasti@gmail.com
}

\begin{abstract}
This mobile implementation study provides a general idea of how existing Athabasca University sites work with the tested mobile devices and identifies the underlying issues as to why they work that way. Factors considered in the implementation include screen size, the use of advanced features, the display of large images, file formats and linking to embedded objects. In the effort to make the sites as mobile-friendly as possible, it is also important to consider what some possible solutions are. Redesigning all those sites carefully, with due consideration to mobile devices, is one possibility considered. This creates a huge burden of site maintenance, as we need to maintain multiple versions of the same page for different devices. Another problem with this approach is that as the capability of mobile devices changes, those sites need to be updated accordingly to reflect the device's capability. This issue is addressed to some extent by creating template-based dynamic pages, and rather than redesigning the pages whenever the device capability changes, one could change the profile of the device.
\end{abstract}

\section{KEYWORDS}

Mobile web sites, screen size, formatting, style sheets, mobile devices

\section{INTRODUCTION}

Athabasca University (AU) is a leading university in Canada, providing open and distance education to more than 38,000 students per year from all over the world. Students can acquire education and degrees without ever having to be physically present at a university campus. This highlights the importance of unconventional but effective and efficient media for providing education and services to students. With the widespread availability of Internet technology, the University is now dependent on the use of the Internet to deliver course materials, to enable students to interact, to provide students with online library access, and to facilitate students in performing administrative tasks such as enrolling into or withdrawing from courses, and even writing exams, remotely.

Originally, AU websites were developed with desktop computers in mind. They have been traditionally designed with the assumption that the user accessing the website has a large, colourful screen and adequate bandwidth for downloading multimedia-rich pages. This assumption cannot be relied on anymore, given the pervasive use of small-screen, low-bandwidth mobile devices as well as the latest $3 \mathrm{~g}$ and $4 \mathrm{~g}$ phones and tablets.

This study investigated the mobile-friendliness of various AU websites and some external sites that are linked from AU sites, specifically journal databases. The websites were tested for visual integrity and functionality retention using less capable mobile devices in order to ensure that students with the less capable phones could still be used if students had not upgraded. It was felt that it is not necessary to test the capacity of the different websites in supporting the more powerful $3 \mathrm{~g}$ and $4 \mathrm{~g}$ phones and tablets because they can (for the most part) display the contents adequately if not better in some cases than on many larger computer screens. The less capable mobile phones have difficulty supporting more sophisticated features such as Java, 
JavaScript, and ActiveX. Some websites provide a fallback mechanism to accommodate these less capable mobile devices; others simply send the web page without considering what kind of device the request came from (Wasti, 2006).

The objective of this study was to evaluate how well the AU websites worked with low bandwidth mobile phones with limited capabilities. The results of the study could then be used to determine how the University can make its websites useful for users with diverse choices of mobile devices. For an open university like $\mathrm{AU}$, it is very important to make its online resources accessible to as wide a range of users and devices as possible.

The M-library project of the AU Library was implemented previously in an attempt to build a platform for AU to develop an effective mobile-friendly library (Cao, Tin, McGreal, et al. 2007). The Digital Reading Room (DRR), Digital Thesis and Project Room (DTPR), Digital Reference Centre (DRC), and AirPAC are some of the outcomes of the project (McGreal, Tin \& Cheung, 2006). These projects formed part of a research focus on mobile learning using stylesheets and proxies (Cheung, McGreal, Tin, et al., 2007) and building a demonstration course specifically for use on mobile phones (Ally, Schafer, McGreal, et al., 2007).

In this investigation, a variety of low bandwidth test devices and a selection of AU websites were studied. Features at the sample websites were tested to see whether they worked as would be expected. There were two key aspects of the test: (1) visual display, and (2) functionality. Some sites rendered well, with their layout intact on small screens, but some features were "crippled" because of the limitations of the underlying device and platform. Similarly, other sites appear relatively deformed but have their features intact. The sites were evaluated for both of the above- mentioned factors.

\section{DISPLAY MODES}

Numerous types of mobile devices capable of accessing the Internet are available. Because of time and resource constraints, it was not possible to test each available device for compatibility with the websites, so for the purposes of this study, three low bandwidth smart phone devices were chosen, each with a different screen size and slightly different browsing characteristics: (1) the HP iPAQ hw6500 (iPAQ), (2) the BlackBerry 8700r (BlackBerry), and (3) the Audiovox SMT5600 (SMT). These devices were chosen specifically because they were low bandwidth older phones. Tablet computers can easily display web sites without formatting or other problems and so were not used, because the purpose of the investigation was to test the sites with the lowest common denominator types of devices.

Using these devices the following modes were generally possible:

1. Single-column mode: In this mode, the web content was presented in a single-column format. It functioned similarly to the BlackBerry but did not use as many optimization techniques. If a table consisted of more than one column, the columns were presented vertically, one after the other. This eliminated the need for horizontal scrolling, but the original page layout was lost, sometimes resulting in pages that were more difficult to read and navigate.

2. Desktop mode: In desktop mode, the page was rendered as if it were being displayed on a desktop computer screen. The sizes of all page elements were kept unchanged. Viewing a page in this mode required significant horizontal and vertical scrolling to view the complete page.

3. Default mode: In the default viewing mode, the relative positioning of page elements was preserved, but the size of the page was proportionally reduced. The width of the resulting page can be larger than the width of the device screen, which required some horizontal scrolling. When trying to proportionally reduce the size of various elements of the page (text, tables, images), the elements sometimes overlapped.

Although some problematic web pages were viewed in different modes to find out whether the problem exists in all viewing modes, the test was primarily conducted in the default viewing mode. The default mode 
is the most likely choice of users and is the one that is likely to produce the best viewing experience for most websites.

There is some variation among the devices used in this study in terms of what web features and file types are supported. Some of the observations in our research represented limitations of the devices themselves and no solutions existed to remedy those limitations; other findings from our study are owing to unavailability of third-party add-on programs for that device's platform. With the exception of DocHawk Platinum and eOffice Professional for BlackBerry, no attempt was made to install third-party software that is only available by purchase. Thus, it is possible that there may be some third-party software programs available for a particular device's platform that would allow opening certain types of files or attachments from that device. Nevertheless, it should only affect the ability to open certain types of files and should have no effect on the HTML page-rendering or the look and feel of web pages when viewed on that device. It is also possible that there may be more advanced web browsers available for those devices that have better page optimization and page-rendering abilities. However, such programs are not easily available or are unavailable for free, which limits their benefit to a small number of mobile device users.

\section{WEBSITE TESTING}

A varied sample of websites was tested. The sites selected were library-related sites, journal databases, hosted journal sites, and some other popular AU sites. Although these sites are mainly library- and journalrelated, the results of this research are general in nature and should be applicable to most university websites.

Tests were performed on the devices by going to various AU websites and assessing them on a scale of $0-3$, where 0 represents not very mobile-friendly, and 3 represents very mobile-friendly. Two factors were considered and assessed accordingly: the visual display of the website as it is rendered on the screen of the device, and the functionality of the page (links, buttons, tabs, navigation, etc.). The following scheme was used in assessing the websites:

Visual display

0 - Page cannot be opened by the device at all.

1 - Page displays on the device with some deformation and/or requires excessive scrolling.

2 - Page displays reasonably well. Some scrolling may be required. Frames and

fixed-size tables may cause some problems.

3 - The page displays perfectly and does not require horizontal scrolling.

Functionality

0 - Page cannot be displayed, or none of the navigation links work.

1 - Only some browsing features work (e.g., many links cannot be opened, or page cannot be

properly navigated).

2 - Most browsing features work (e.g., most links, buttons, and navigation items work).

3 - All links and all features work. Form submission and buttons, and so on, all work.

\section{TEST RESULTS}

Flash player presented a significant problem as any site using Flash would not display properly on the majority of phones. The phones also generally did not support a PDF reader, so trying to open a PDF file from any website was problematic. Such limitations are considered the limitations of the device itself and not shortcomings of the website. However, if a site primarily relied on the device being able to make use of those features, the mobile-friendliness grading of that site was reduced accordingly.

The sample pages showed the following results 
Athabasca University Home Page http://www. athabascau.ca/

The BlackBerry had a default single-column view and displayed the AU home page well. The PPAQ and SMT could also display the page in single-column format that was similar to the display in the BlackBerry but looked less polished. The default mode in the IPAQ and SMT required horizontal scrolling, and the page looked slightly deformed. Regardless of the display mode, however, all navigation links worked well on all three devices.

\section{myAU $h t t p: / / m y . a t h a b a s c a u . c a$}

The iPAQ and SMT both display this site fairly well, and all navigation links and site logins worked as expected. On the other hand, the BlackBerry could not open this site at all. It generated a dialogue box: "HTTP error 406: Not Acceptable". This appears to be caused by a configuration problem on the server side; the server incorrectly assumed that the client device was unable to render the page and so did not send any content.

Online Registration https://tux.athabascau.ca/oros/jsp/welcome.jsp

This main registration site rendered well in all three devices. All links and form submissions worked as expected.

AU Intranet $h t t p: / /$ intra. athabascau.ca/

The BlackBerry rendered this site fairly well in its one-column layout. In the default viewing mode of the iPAQ and SMT, extensive horizontal scrolling was required and navigating was more difficult. The excessive horizontal scrolling was made necessary by the use of fixed-size tables. Setting up vacation notices and changing forwarding email addresses worked well in all three devices.

AU Webmail https://secure. athabascau.ca/webmail

Webmail worked well with the PAQ and BlackBerry but did not open in SMT because of frames.

Research Centre $h t t p: / / w w w . ~ a t h a b a s c a u . c a / r e s e a r c h$

The Research Centre site rendered well in the single-column layout of the BlackBerry and fairly well in the default layouts of the iPAQ and SMT. The site used fixed-size tables, so it requires a lot of horizontal scrolling. All links on the page worked well.

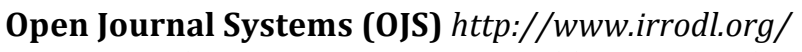

Open Journal Systems (OJS) was tested by going to the journal site of the International Review of Research in Open and Distance Learning (IRRODL). This site rendered well in the BlackBerry and in the singlecolumn format of the iPAQ and SMT. However, it rendered rather poorly in the default layout of the iPAQ and SMT: two columns of the table almost overlapped. Editing and saving submissions worked well, but files could not be uploaded through any of the tested devices.

Open Conferencing System (OCS) http://tools.elab. athabascau.ca/tools/open-conference-system This site rendered very well on all three devices. Single-column and default views all worked well. All links on the site also opened nicely. The file-upload feature on the paper submission page was the only part of the site that did not work. Public Knowledge Project's Open Conferencing System (OCS) worked as the back end of the paper submission system; so the file-upload problem was determined to be with OCS rather than with the OCS site.

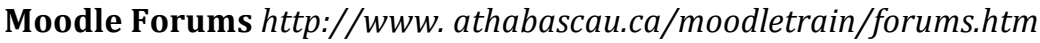

The Moodle forum site displayed well on all three devices. Posting, reading, and editing messages worked well. As with other sites that required file uploads, none of the devices were able to upload files from this site.

AUSpace $h t t p: / / a u s p a c e$. athabascau.ca/

The AUSpace site displayed well in the BlackBerry and in the single-column layout of the PAQ and SMT. The default layout of the PAQ and SMT required horizontal scrolling because of tables. Searching and 
viewing files worked well in all devices. However, when trying to submit articles to AUSpace, it was not possible to upload files because the input box for the filename and browse button were missing in all three devices. This was not a problem of the site itself but rather a limitation of the mobile devices.

\section{AU Library Sites}

Main Site - http://library. athabascau.ca/

Digital Reading Room (DRR) - http://library. athabascau.ca/drr

Digital Thesis and Project Room (DTPR) - http://library. athabascau.ca/

DTPR Digital Reference Centre (DRC) - $\underline{\text { http://library. athabascau.ca/drc }}$

All AU Library sites tested were found to be very mobile-friendly. The DRR, DTPR, and DRC used similar layouts that are fluid, allowing a smooth flow of text. As those sites did not use fixed-size tables, the display was consistent and predictable across all the devices. AU Library sites adapted to the client device for optimal mobile-friendliness without sacrificing the richer web content. This was done by detecting what type of device was accessing the pages and then sending the appropriate version of the web page to the device.

The AU Library has integrated the mobile conversion services Google Mobile, Skweezer, and IYHY into some of the reading resources in DRRs. These third-party services work as proxy servers to provide suitable formatting of existing websites for mobile devices (Athabasca University Library, 2011).

\section{AU Library Catalogue: AirPAC http://aupac.lib. athabascau.ca/airpac/}

This site is a mobile-optimized version of the AU Library catalogue. AirPAC formats its response for the type of device that is used to access the site. It sends a smaller version of the page to the SMT and BlackBerry to accommodate the small screen area, while it sends a larger version to the iPAQ with more screen area available. The site displayed very well in all three devices and in all view modes, and all links from this site worked flawlessly.

\section{Journal Databases}

An extensive test was performed on all journal databases linked from the AU Library website. The journal databases were assessed on a scale of $0-3$, where 0 represents not very mobile-friendly, and 3 represents very mobile-friendly. The grading scheme used for assessing the journal databases was the same one used for evaluating the other AU websites listed in Table 3. However, for the databases, the visual display and web functionally were considered together and graded accordingly. More than 120 databases were examined and their scores ranged from 0 to 3 on all devices, with varied mixes of accessibility, some being high with one device and low with another.

\section{DISCUSSION OF RESULTS}

Based on this study's test results of the AU websites and the journal databases, the following visual display and functionality issues were identified as affecting the mobile-friendliness of the sites tested:

1. Some pages were displayed with unnecessary spaces at the top of the pages and large gaps in the middle. This is normally caused by the use of complex structures in the HTML page design, such as tables, nested tables, transparent pictures used for layout, and so on. When the mobile browsers try to optimize such pages to fit a small screen, the page may be deformed in many ways, depending on the optimization method used by the browsers.

2. If a page contains fixed-width blocks, excessive scrolling is necessary when viewing through mobile devices.

3. Flash-dependent websites pose another challenge for mobile browsers. Some websites depend on Flash for navigation menus. It is impossible to successfully visit such sites through devices that do not support Flash (e.g. iPhone, iPad).

4. Because the mobile devices only partially support JavaScript, the result of visiting a JavaScriptdependent site is unpredictable. Some features may work; others may not. For example, some sites extensively use JavaScript to open new browser windows. Such links almost always fail to open in 
the tested mobile devices. Other sites have entirely JavaScript-dependent navigation menus. In such cases, it is not possible to visit the pages linked from the menus.

5. Extensive use of specialized file formats - such as PDF, e-book, Microsoft Word, and PowerPointcan be problematic for some mobile devices. Some browsers do not support opening those file types, so sites making heavy use of those file formats would not be friendly to these devices.

6. The use of frames also reduces the mobile-friendliness of the site. Different mobile devices support frames to different degrees. The iPAQ does not have a problem with frames, whereas the SMT cannot open horizontal frames. The BlackBerry linearizes the frames and displays them one at a time.

7. Some web servers make assumptions about the device accessing the website. For example, in one instance, the BlackBerry could not open a site because the server refused to send content; the server assumed that the device could not render the file.

8. One major problem common to all devices is the inability to upload files. None of the tested mobile browsers allowed files to be uploaded because they could not recognize the file browsing buttons.

The following chart (Figure 1) provides a mobile-friendliness score, which is the sum of the scores assigned to the different sites for individual devices. For example, adding the scores of the three devices used, the total score for the AU home page would be 14 out of the maximum 18. These scores were then normalized for each site to a maximum 100 points. Thus, the normalized score of the AU home page would be 77.77 points out of a maximum 100. The chart should be interpreted this way: "Two of the tested sites scored higher than 90 , five sites scored between 80 and 90, five sites scored between 70 and 80 ," and so on. As we can see from the chart, in general, most AU websites are quite mobile-friendly

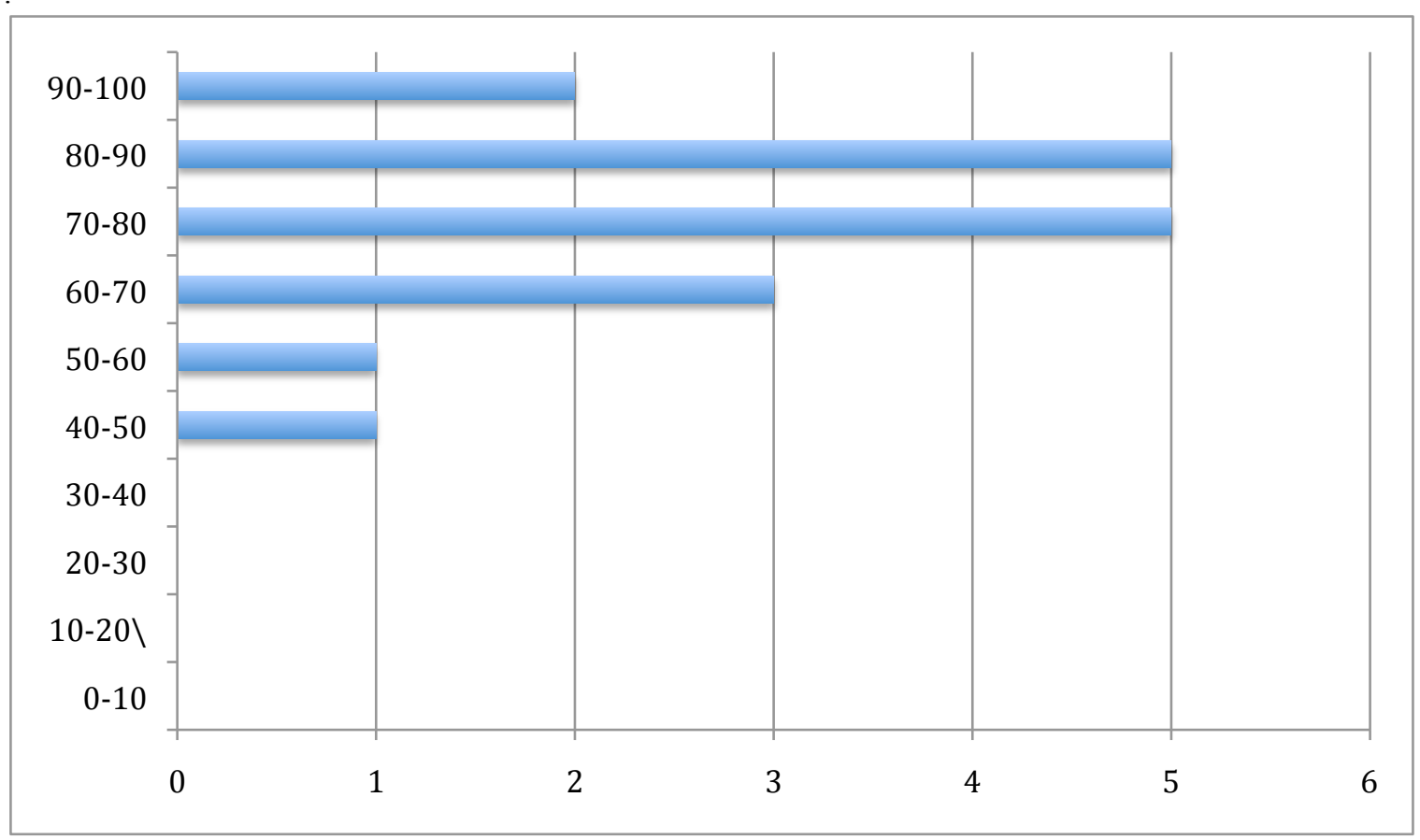

Figure 1. Histogram of mobile friendliness of tested AU websites

A similar chart (Figure 2) provides a mobile-friendliness score for the more than 120 journal database sites linked to the AU Library website. The majority of websites scored 60 or higher in terms of mobilefriendliness. Some sites were problematic for the mobile devices, and scored lower than 50, so in general the external sites were not as mobile-friendly as the AU websites. 


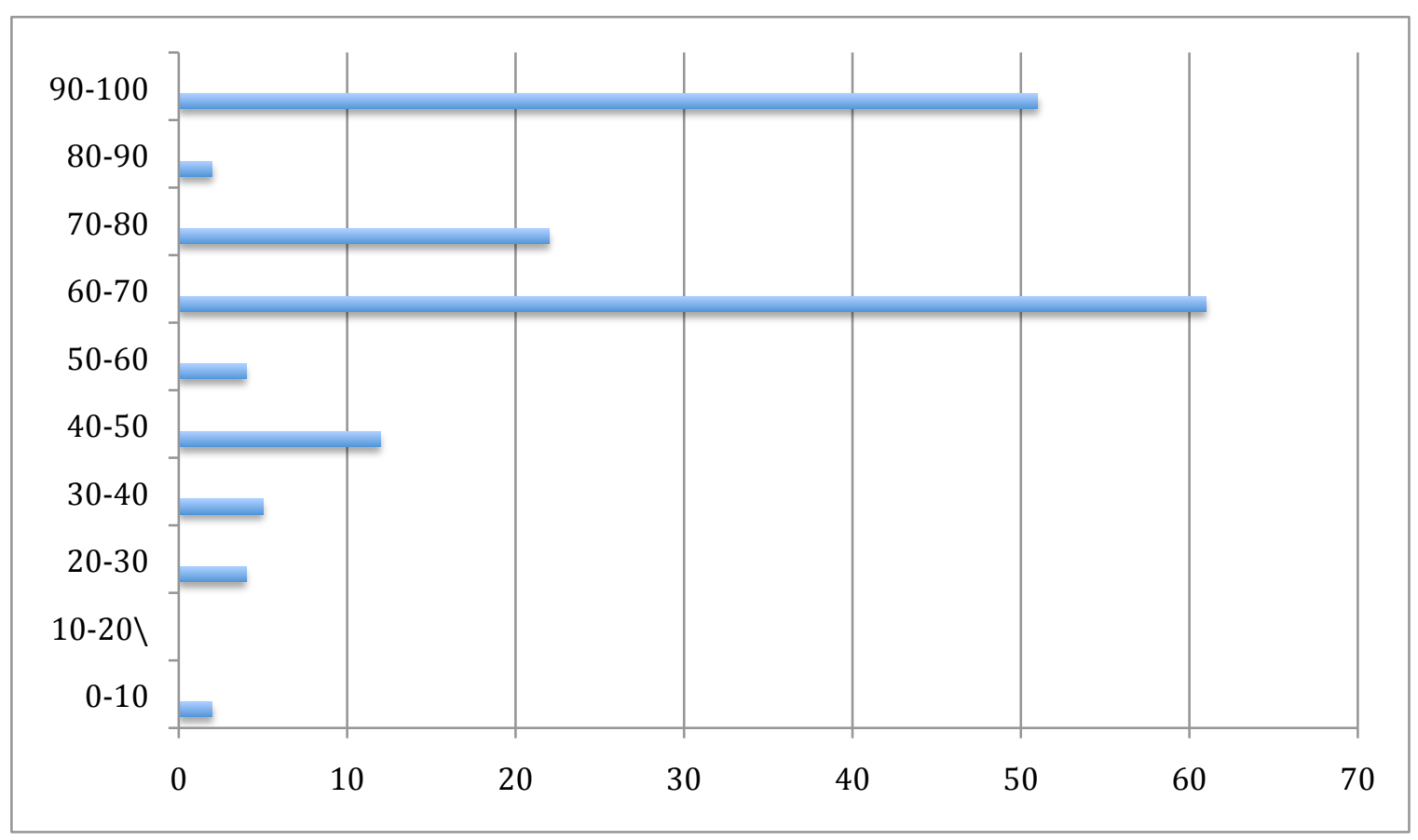

Figure 2. Histogram of mobile friendliness of journal databases

Nevertheless, overall, the websites and journal databases were found to be quite mobile-friendly. This study shows that most AU websites are viewable and operable from the tested low bandwidth mobile phones. Some sites did not retain their layout and visual design when they were viewed from the test devices but were still functional. Some sites could be rendered nicely but lost some functionality, such as their navigation links and some JavaScript-dependent features. Some sites (especially the AU Library ones) were almost perfect visually and functionally when accessed from the smart phones.

This study determined that most of the AU Library websites (the main site, DRR, and DTPR) were designed with specific attention to the requirements of mobile devices: the server sent responses custom-tailored to the device being used to access the sites. The benefit of this approach is that mobile device users can conveniently enjoy their device's resources at the same time as desktop users can make full use of their respective capabilities, receiving multimedia-rich content and more advanced graphical display. The downside is the need to maintain different versions of the same web page for different device profiles.

\section{CONCLUSION AND RECOMMENDATIONS}

Whether to characterize a website as mobile-friendly is not a simple yes or no question. There are variations in the degree to which various sites are friendly to mobile devices. Making a general statement about a website being mobile-friendly or mobile-unfriendly is not always accurate. There are so many different mobile hand-held devices with differing feature sets that it is difficult to make a generic statement about a website-while at the same time covering all possible devices and browsers that may access that site. The objective of this study was not to pronounce various sites as "mobile-friendly" or "not friendly," but to get an overall picture of how the sites are visually displayed and function when viewed through the hand-held devices that were used for testing.

The capabilities of mobile devices are also rapidly changing. Manufacturers of those devices continuously add new features, and software developers develop more capable and "smart" software solutions to overcome 
some of the inherent limitations of those devices. For example, even if this study found that a device currently does not support native viewing of PDF files, that capability has since been added in the more recent models, making these findings somewhat obsolete. Thus, the direct results stated in this study are time-sensitive; that is, their accuracy is valid only for the current time and only for the specific models of those devices. Even so, despite the time- and device-sensitive nature of the results, we can draw some conclusions as to what factors contribute to making a website more mobile-friendly or less mobile-friendly:

1. Screen size considerations. One of the main constraints of small devices such as smart phones is the screen size. So, any web page that relies on various HTML elements being displayed in a fixed size is bound to cause problems on small-screen devices. To avoid such problems, it is recommended that the use of fixed-size tables be minimized or eliminated altogether. The positioning of HTML elements should be relative as opposed to absolute, so that when the page is resized and viewed on small screens, its layout remains intact.

2. Careful use of advanced HTML features. Many smart phones provide only partial support for Cascading Style Sheets (CSS) and JavaScript. Thus, if a web page is intended to be viewed on both desktop computers and small-screen low bandwidth mobile devices, the page integrity can be preserved on the mobile devices by limiting the use of CSS and JavaScript features to those that are supported by both.

3. Large images. Avoiding the use of large images in websites helps to make the site friendlier to mobile devices. When a large image is rendered on a small screen, the device may either reduce the size of the image to fit the screen, or keep the size of the image unchanged, which then causes the need for excessive scrolling. If a large image is resized, important details of the image may be lost, defeating the purpose of the image. This is especially problematic if an image map is used for page navigation. If the image is not resized, the required excessive scrolling makes it harder to navigate the page.

4. File format of the web content. Excessive use of file formats that are not supported by mobile devices also makes the site less friendly to mobile devices. Most devices are capable of viewing simple HTML pages, but they may not be able to open other types of media, such as PDF files, PowerPoint files, videos, or Flash content. In such a case, even if the device is able to access some pages of that website, it cannot fully take advantage of the materials and links provided.

5. Embedded objects. Special care should be taken when embedding objects on websites. Embedding objects such as audios, videos, and Flash in a web page enriches the page for desktop computer users, but it assumes that all browsers accessing the site are capable of handling those embedded objects. This is a precarious assumption in terms of the access of mobile devices. Mobile browsers usually disregard embedded objects. Therefore, if the embedded object is a crucial part of the page, the rest of the page may not make sense to the mobile device user. Instead of embedding an object on the web page, it is generally better to include a link to such a file. That way, the user can download the file and open with a third- party program, if such a program exists. Even if the user chooses not to download the file, the web page integrity remains intact.

This study provides a general idea of how existing AU sites work with the tested mobile devices and identifies the underlying issues as to why they work that way. However, in the effort to make the sites as mobile-friendly as possible, it is also important to consider what some possible solutions are. Redesigning all those sites carefully, with due consideration to mobile devices, is one possibility, but it is a very impractical one. The cost of resources associated with this route can be enormous. Another possibility is creating a mobile-friendly version of each AU web page and serving those pages instead of the regular pages whenever mobile devices make page requests. This creates the huge burden of site maintenance, as we need to maintain multiple versions of the same page for different devices. Another problem with this approach is that as the capability of mobile devices changes, those sites need to be updated accordingly to reflect the device's capability. However, this issue can be addressed to some extent by creating template- based dynamic pages, and rather than redesigning the pages whenever the device capability changes, one could change the profile of the device, which in turn would be reflected in the dynamic page.

A totally different approach that is gaining some popularity is the use of an intermediary proxy- like adaptation layer for the web content. That way, there is no need to maintain multiple versions of the same 
website, and it also does not require redesigning existing websites. In effect, whenever a user makes requests to a web page, the request can be routed through an intermediary service that identifies the requesting device, gets the page from the web server on behalf of the device, and reformats the page - thus making it suitable for that particular device - and then passes it on to that device. The success of such an approach depends entirely on the capability of the intermediary service. One advantage of this approach is that the burden of making the web pages user-friendly now shifts to the intermediary service from the web server or the web page administrator. Another advantage is that end users do not need to make any special adjustments or install special software on their devices.

There are some websites that provide such intermediary services. Skweezer http://www.skweezer.net/, IYHY (http://www.iyhy.com/ , and Google Mobile http://www.google.ca/mobile/ are examples of such services. The AU Library is experimenting with the integration of those services with some of the Digital Reading Room resources. Few AU sites employ such service at this time. In the testing, most sites that required login/authentication could not be viewed through those services. However, when those services mature and become more capable, they might make mobile web access much more comfortable.

\section{REFERENCES}

Ally, M., McGreal, R., Schafer, S., Tin, T., \& Cheung, B. (2007). Use of mobile learning technology to train ESL adults, Sixth International Conference on Mobile Learning Available from http://www.cclcca.ca/CCL/Research/FundedResearch/201009AllyMcGrealSchaferTinCheung.html

Athabasca University Library. (2011). AU Digital Reading Room Home (integrated mobile conversion services) Retrieved from http://library.athabascau.ca/drr/mobile2.php? course $=$ mba\&id $=441 \&$ sub $=5$

Cao, Y., Tin, T., McGreal, R., Ally, M., \& Schafer, S. (2007, November). Building an effective mobile-friendly digital library to support mobile learners: A case study of the Athabasca University M-library Project. Paper presented at the First International M-Libraries Conference, Milton Keynes, UK.

Cheung, B., McGreal, R., \& Tin, T. (2007, July). Implementation of mobile learning using smart phones at an open university: From stylesheets to proxies. Paper presented at the IADIS Mlearn 2007 Conference, Lisbon, Portugal.

McGreal, R., Tin, T., \& Cheung, B. (2006). Digital media at Athabasca University - Canada's Open University - Going Mobile. International Council for Distance Education (ICDE) 2006 Retrieved .from http://www.icde.org/oslo/icde.nsf/CEFB5A35F7FC2EF8C1257298004C8616/\$FILE/461.pdf

Wasti, R. (2006, March). A study of the mobile-friendliness of selected Athabasca University websites. Available from http://library.athabascau.ca/drr/Paper/final web.pdf 